\title{
Perceptual learning in pattern discrimination tasks with two and three schema categories'
}

BILL R. BROWN and SELBY H. EVANS, Texas Christian University, Fort Worth, Tex. 76219

This research demonstrated that humans can develop the ability to distinguish between stimuli of either two or three schema families without externally administered knowledge of results or prior familiarization with the population prototypes. These demonstrations of schematic concept formation support the perceptual learning theory that differentiation of higher order variables can occur on the basis of information derived from perceiving the stimuli, and indicate the need for further investigation of the detailed conditions under which the phenomenon occurs.

A number of investigators (Attneave, 1957; Oldfield, 1954; Woodworth, 1938) have suggested that the encoding of redundant or schematic aspects of stimuli reduces information processing and storage requirements. A schema is assumed to be abstracted as a set of commonly occurring characteristics in a collection of otherwise different instances; Evans \& Edmonds (1966) thus have pointed out that a schema may be regarded as a type of higher order variable. Correspondingly, a population of stimuli, all of which can be described by a schema rule, constitutes a schema family.

The natural environment typically provides a collection of mixed stimuli belonging to different schema families. In this context, a number of studies (Brown, Walker, \& Evans, 1968; Edmonds, Mueller, \& Evans, 1966; Evans, 1964; Evans \& Arnoult, 1967; Rosser, 1967) have demonstrated that humans can develop the ability to distinguish between stimuli of different schema families without externally administered knowledge of results or prior familiarization with the population prototypes. Evans (1967a) has termed this learning process schematic concept formation (SCF). These demonstrations of SCF tend to support the perceptual learning theory of the Gibsons (Gibson, 1959; Gibson \& Gibson, 1955) in that differentiation of higher order variables (i.e., schemata) occurred only on the basis of information derived from preceiving the stimuli.

The implications of the SCF process for a theory of perceptual learning rests upon the supposition that such learning occurs in tasks which have perceptual and memory requirements representative of the natural environment. The research reported here, using a same-different task (Gibson \& Gibson, 1955), was intended to demonstrate that humans can develop the ability to distinguish among stimuli sampled from either two or three schema families. Moreover, the only information available to the Ss was that derived from perceiving the stimuli themselves. The first study used stimuli sampled from two different schema families, whereas the subsequent experiment required discriminations to be made among instances from three schema families.

\section{EXPERIMENT Subjects}

The Ss were 81 naive undergraduates enrolled in introductory psychology courses at Texas Christian University. Stimuli

The VARGUS 7 computer program (Evans, 1967b) was used to generate histoform stimuli which were $50 \%$ redundant. Constraint redundancy (Evans, 1967c) refers to the extent to which a population of stimuli adhere to a schema rule. The instances were 24 columns in length and were produced from a seven-element Markov process by converting the elements into column heights. The program introduced the schemata into the stimuli by selecting column heights with transitional probabilities favoring three different most probable sequences (MPSs). The stimulus populations sampled in the present research can be found in Bersted, Brown, \& Evans (1968) and are there designated as Schemata 2,3 , and 4 .

The manner in which the stimuli were generated assured that corresponding instances of the three schema families had the same variance. This variance measure is the proportion of schematic steps (POSS) for each instance and it describes the adherence of each individual instance to the MPS. The POSS statistic has been discussed in detail by Bersted, Brown, \& Evans (1968). Procedure

The Ss were given 60 trials by means of Xeroxed booklets. On a typical trial, the Ss viewed two histoform stimuli and then judged them to be examples of the same pattern or of two different patterns. The Ss were allowed $10 \mathrm{sec}$ per trial to study the stimuli and to mark their answers. No knowledge of results or other external reinforcement was provided.

As stated previously, this experiment involved only two schema families. Fifty per cent of the 60 trials in this task involved pairing of instances from Schema 2 and Schema 3. The correct response to each of these pairings was "different." Another 25\% of the trials involved pairing of instances from Schema 2, while the other $25 \%$ consisted of pairs of instances from Schema 3. The correct response to each of these pairings was "same," since any two instances presented to the Ss were sampled from the same schema family.

No one instance from either Schema 2 or 3 was presented more than once during the 60 trials. The two instances presented on each trial were paired on the basis of their having equal POSS values. This procedure assured that the instances of any given pair were equal in difficulty as far as abstraction of the schema rules was concerned.

Three stimulus presentation orders were used in this experiment. Each of the orders was random except for constraints which insured a homogeneous mixing be tween the booklet pages containing stimuli of the same schema family and those bearing stimuli of different schemata. The task was administered to three Ss per experimental session and each of the three $S s$ received a different order of presentation.

\section{EXPERIMENT 2}

This experiment was identical to the first study in all but two respects. First, a new group of 72 naive $S$ s participated in this study. These Ss were enrolled in introductory psychology courses at Texas Christian University. Second, this study required discrimination among instances sampled from three different schema families, whereas the first experiment involved two schemata. Fifty per cent of the 60 trials involved the pairing of instances from different schema families (i.e., Schemata 2, 3 , and 4). Each of the remaining $50 \%$ of the trials consisted of two instances sampled from the same schema family, Schemata 2 , 3 , and 4 each being represented on 10 trials. The three schema families were represented an equal number of times across the 60 trials. Each instance was presented only once in the task. As in the first study, the instances of any one pair had equal POSS values and three orders of stimulus presentation were used.

\section{RESULTS AND DISCUSSION}

A demonstration of SCF in the samedifferent task requires that the $S$ s make a "different" response when viewing two instances of different schemata and a "same" response when viewing two instances of the same schema family. The task thus demands the developmer $t$ of the ability to assign patterns to their corresponding schema families on the basis of the 


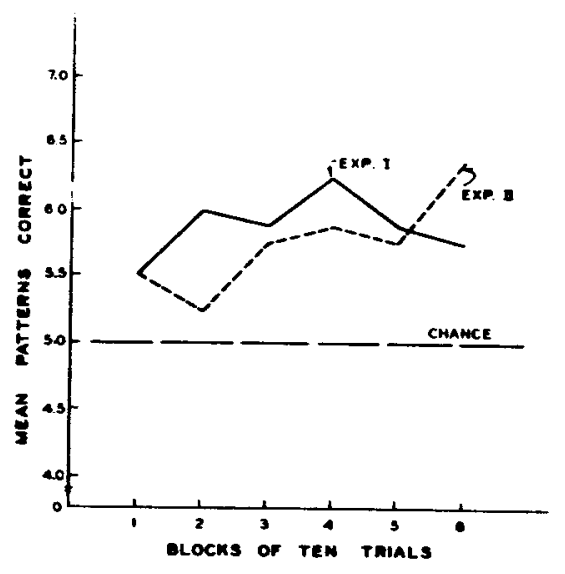

Fig. 1. Mean correct responses over six blocks of 10 trials for the $78 \mathrm{Ss}$ in the two-echemata task (Experiment 1) and the 70 Ss in the threeschemata task (Experiment 2).

information derived from perceiving the patterns themselves. In the two experiments reported here, a S's responses were considered to be consistent with the schema-defined classes if 13 correct discriminations were made during the last 20 trials of the same-different task. A table of the cumulative binomial distribution (Harvard University Computation Laboratory, 1955) shows that 13 successes will occur by chance over 20 trials with a probability of .131

The first experiment used the samedifferent task in which only two schema families were represented. The data from three human Ss were discarded in this study due to their failure to understand the task instructions. Each of these Ss made a "D" response on each trial; two of the three Ss made no attempt at studying the patterns before responding. Similarly, the data from two $\mathrm{Ss}$ in the second study were discarded for the same reasons.

In the first experiment, $31 \%$ of the $78 \mathrm{Ss}$ satisfied the criterion of consistency. These Ss did in fact exhibit discriminatory responses consistent with the schema rules despite the lack of information as to the appropriate schema categories. In the second study, on the other hand, $49 \%$ of the $70 \mathrm{Ss}$ met the criterion of consistency. Contrary to expectation, the proportion of Ss responding in a manner consistent with the schema rules in the three-schemata task was thus larger than the corresponding proportion in the two-schemata experiment. Figure 1 shows that, except for the last block of 10 trials, performance for all Ss in the two-schemata task was higher than that observed in the second study.

The fact that a greater proportion of Ss in the three-schemata experiment satisfied the consistency criterion, in comparison to the two-schemata task, indicated that more

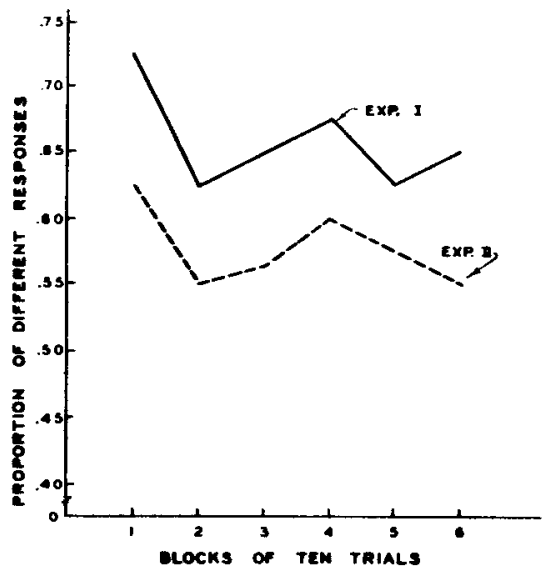

Fig. 2. Proportion of different responses over six blocks of 10 trials for the $78 \mathrm{Ss}$ in the two-schemata task (Experiment 1) and the $70 \mathrm{Ss}$ in the three-schemata task (Experiment 2).

detailed response trends required evaluation. Specifically, the question was raised as to whether or not the two groups differed with respect to the proportions of "different" and "same" responses exhibited across trials. Figure 2 indicates that in both experiments the Ss showed a strong bias towards making "different" responses. The Ss in the three-schemata task, however, made a smaller proportion of such responses than did the Ss in the first experiment.

The above differences in response trends suggest several points which may be relevant to the observation that a greater proportion of Ss met the consistency criterion in the three-schemata study.

First, the fact that the $S s$ in both experiments made a majority of "different" responses suggests that more categories than there were schema families were being used. This suggestion receives support from an experiment currently being conducted in which $S s$ are being required to actually sort patterns with no information as to the number of schema families represented in the task. The preliminary results indicate that the Ss use more categories than are actually needed for correct sorting of the patterns into schema-defined categories (i.e., several categories may reflect each schema separately). It is important to note that in the two same-different tasks reported here, the Ss were not told the number of schema families represented in the task. There is thus the possibility that these Ss were implicitly using several categories for each schema. To the extent that the resulting response bias towards making "different" responses is greater in a two-schemata task, as compared to the three-schemata experiment, discrimination performance would likely be poorer in the former.

Second, Evans (1964) has pointed out that the performance of Ss meeting the consistency criterion is characterized by low rates of information acquisition, and that such gradual performance increments cannot be unequivocally interpreted as poor performance. Rather, it might be interpreted as a sampling process which insures that the final result will be based on a large sample by taking a small amount of information from each instance. There is thus the possibility that the presence of three-schema families in the task encouraged such a sampling strategy and tended to increase the possibility of the Ss meeting the consistency criterion.

Finally, there remains the possibility that the $S$ samples for the two experiments differed with respect to the degree to which instructions were interpreted correctly, amount of involvement in the task, and the numerous other variables which might affect performance. This alternative does not seem likely, however, in view of the consistency of performance observed in these experiments and that obtained in other tasks which were discussed previously. What is needed here, of course, is a study using a design which permits direct statistical comparisons between performance under the two- and three-schemata conditions.

The experiments reported here have demonstrated that some humans can develop the ability to distinguish between stimuli of either two or three different schema families. Since the natural environment usually provides mixed instances of numerous schema classes, the SCF process thus represents a means by which humans can effectively reduce environmental complexity. Moreover, since the environment seldom provides schema prototypes, the occurrence of SCF without prior familiarization with population prototypes demonstrates that schema learning may be useful in the construction of perceptual learning theories. The results reported here do in fact support the theory that differentiation of higher order variables can occur only on the basis of information derived from perceiving the stimuli.

Determination of the exact implications of the SCF process for perceptual learning theories requires specification of the detailed conditions under which the phenomenon occurs. The studies reported here have raised several questions with respect to the factors influencing $\mathrm{SCF}$, and thus indicate a need for further empirical research concerning the effects of constraint redundancy, types of instructions, and the appropriateness of different types of response measures.

\section{REFERENCES}

ATTNEAVE, F. Transfer of experience with a class-schema to identification-learning of patterns and shapes. Journal of Experimental Psychology, 1957, 54, 81-88. 
BERSTED, C. T., BROWN, B. R., \& EVANS, S. H. A standard set of VARGUS 7 patterns at three levels of schematic redundancy. Psy chonomic Monograph Supplements, 1968, 2 (13, Whole No. 29), 251-282.

BROWN, B. R., WALKER, D.W., \& EVANS, S. H. Schematic concept formation as a function of constraint redundancy and knowledge of results. Psychonomic Science, 1968, 11, 75-76.

EDMONDS, E. M., MUELLER, M. R., \& EVANS, S. H. Effects of knowledge of results on mixed schema discrimination. Psychonomic Science, $1966,6,377-378$

EVANS, S. H. A model for perceptual category formation. Unpublished doctoral dissertation, Texas Christian University, 1964.

EVANS, S. H. A brief statement of schema theory. Psychonomic Science, 1967a, 8, 87-88.

EVANS, S. H. VARGUS 7: Computed patterns from Markov processes. Behavioral Science, 1967b, 12, 323-328.

EVANS, S. H. Redundancy as a variable in pattern perception. Psychological Bulletin, 1967c, 104-113.

EVANS, S. H., \& ARNOULT, M. D. Schematic concept formation: Demonstration in a free sorting task. Psychonomic Science, 1967, 9, 221-222.
EVANS, S. H., \& EDMONDS, E. M. Schema discrimination as a function of training Psychonomic Science, 1966, 5, 303-304.

GIBSON, J. J. Perception as a function of stimulation. In S. Koch (Ed.), Psychology: $A$ study of a science. Vol. 1. New York: McGraw-Hill, 1959.

GIBSON, J. J., \& GIBSON, E. J. Perceptual leaming: Differentiation or enrichment? Psychological Review, 1955, 62, 32-41.

HARVARD COMPUTATION LABORATORY. A table of the cumulative binomial Harvard University Press, 1955.

OLDFIELD, R. C. Memory mechanisms and the theory of schemata. British Journal of Psychology, 1954, 45, 14-23.

ROSSER, E. M.Categorization and discrimination of tone sequences. Unpublished doctoral dissertation, Harvard, 1967.

WOODWORTH, R. S. Experimental Psychology. New York: Holt, 1938. NOTE

1. This research is a part of the first author's doctoral dissertation submitted at Texas Christian University in 1968. Support for the research was provided by a Department of Defense Project THEMIS (DAADO5-68-C-0176) under the Department of the Army.

\section{Sequential blanking and visual form perception'}

A. BELL, R. G. FORSTER, ${ }^{2}$ F. J. FINNEGAN, ${ }^{3}$ M. KATZ, J. I. La SUSA,4 and M.S. MAYZNER, New York University, Bronx, N.Y. 10453

The present study replicates and extends previous findings by Julesz, in which he studied sequential and nonsequential presentation orders for the sides of variously shaped polygons. Our results fully confïm his findings and further suggest that his results are but still another instance of sequential blanking effects in man's visual information processing system.

In a recent paper, Julesz (1967) studied visual form perception for various polygons by presenting the sides of the polygons, which were thin bright slits on a black background, cyclically in sequential and nonsequential orders, at various display rates. The Ss were requested to indicate when the polygon in question was perceived as a single Gestalt, i.e., all sides joining and appearing to occur simultaneously to $S$. The results clearly showed that for $S$ to report perceiving the polygon in question as a single Gestalt, the display time per side needed to be about 5 to $10 \mathrm{msec}$ lower for the nonsequential presentation order than for the sequential presentation order. Julesz (1967) conceptualizes his findings in the following terms: "One recognition mechanism might be based on scanning sequential ly each side of the polygon and noting the temporal order of vertices where the jump from one set of orientation detectors to another occurs. A conceptually simpler but actually more complex model might be based on parallel organization. Here each possible slit detector combination forms a complex unit which might simultaneously process each side of the polygon in question [pp. 139-140]." Julesz (1967) concludes: "Since in the experiments for both the sequential and nonsequential presentation the same perceptual criterion of seeing a Gestalt was used, we might expect the polygon detector would be insensitive to the temporal order of the occurrence of edges. This expectation is in disagreement with the findings [p. 141]." Thus, it would appear from Julesz's results that the parallel organization hypothesis does not yield the expected results and that sequential organization processes predominate.

Since Julesz's finding has considerable theoretical importance for visual form perception, it was decided to replicate his study, using a computer-based cathode-ray tube (CRT) display system, with a larger variety of polygons and to employ a slightly different psychophysical procedure, as well as the one employed by Julesz. In addition, Julesz incidentally noted ${ }^{5}$ that in the nonsequential order, $\mathrm{Ss}$ reported seeing sides missing from the polygon prior to perceiving it as a single Gestalt, and we were particularly interested in seeing whether this finding could be replicated and whether it might not be another instance of our apparently new perceptual phenomenon of "sequential blanking" (Mayzner, Tresselt, \& Cohen, 1966; Mayzner, Tresselt, \& Helfer, $1967 \mathrm{a}, \mathrm{b})$.

\section{SUBJECTS}

Five male Ss were employed for the main study and an additional 14 Ss were exposed to certain critical display configurations in a subsequent group demonstration of the effects which were found in the main study. APPARATUS

The eight pairs of polygons that were studied were presented on two Fairchild CRT display consoles simultaneou sly, allowing two Ss to be tested simultaneously. Both Fairchild CRT display consoles were slaved to a 340 Master Display, driven by a PDP.7 digital computer. A complete description of this system may be found in our previous papers (Mayzner, 1968; Mayzner, Tresselt, \& Helfer, 1967a)

\section{MATERIALS}

The stimulus materials consisted of the five polygons employed by Julesz (1967), i.e., a pentagon, a hexagon, and a triangle, but with each side divided in half, so that six sides were displayed, either sequentially or nonsequentially, and a second pentagon and triangle, in which the vertices or vertices and part sides were displayed, either sequentially or nonsequentially, and labelled as in Julesz's paper, A, B, C, D, and E, respectively. In addition, three new polygons were added, labelled $F, G$, and $H$, respectively, and consisted of a very irregular 12-sided polygon, a square, and a very irregular sixsided polygon. Each of the eight polygon types, A through $\mathrm{H}$, was presented on the CRT in pairs, side by side, with the sides of one of the pairs of polygons presented sequentially, while 1 in. to the right an identically shaped polygon was displayed, but with its sides presented in a nonsequential order, following Julesz's orders of side presentation exactly, for his polygons, A through $E$, and employing a random nonsequential order of side presentation for Polygons $F, G$, and $H$. Each polygon was approxi- 